\title{
Experimental study on axial development of bubbly flow under normal- and micro-gravity environment
}

\author{
Naohisa TAMURA, Norihiro FUKAMACHI, Tatsuya HAZUKU \\ Tomoji TAKAMASA, Takashi HIBIKI
}

\begin{abstract}
In view of the great importance of two geometrical parameters such as void fraction and interfacial area concentration to the accurate two-phase flow analysis at microgravity conditions, axial developments of flow parameters such as void fraction, interfacial area concentration, bubble Sauter mean diameter, and bubble number density were measured by image-processing in bubbly flow at microgravity and low liquid Reynolds number conditions where the gravity effect on the flow parameters were pronounced. Negligible bubble breakup was observed because of weak turbulence under tested flow conditions. The velocity profile entrainment effect under microgravity was likely to be comparable to the wake entrainment effect under normal gravity in the tested flow conditions.

Keywords: Microgravity, Interfacial area concentration, Drift-flux model, Multiphase flow, Bubbly flow
\end{abstract}

\section{Introduction "}

Recently, breakthrough analytical and experimental studies at microgravity conditions were performed to address the effect of the gravity on the bubble dynamics. Tomiyama showed that the relative velocity between a single bubble and a liquid flow in a confined channel was derived by taking into account the effect of a frictional pressure gradient due to a liquid flow [1]. This analysis implied that the frictional pressure gradient due to the liquid flow might be regarded as an additional source of buoyancy and the effective body acceleration would be expressed by the linear superposition of flow-induced body acceleration and effective body acceleration. On the other hand, Hibiki inspired the detailed formulation of the $\mathrm{drift}$ velocity considering the wall shear stress [2], which can be applied to microgravity conditions [3].

In view of the great importance of two geometrical parameters such as void fraction and interfacial area concentration to the drift velocity model and accurate two-phase flow analysis at microgravity conditions, the present study is aiming at the measurements of the axial developments of flow parameters such as void fraction, interfacial area concentration, bubble Sauter mean diameter, and bubble number density at microgravity and low liquid Reynolds number conditions where the gravity effect on the flow parameters are more pronounced. In the experiments, the flow measurements were performed by the image processing method with three digital video cameras placed at $z / D$ 
$(D=9.0 \mathrm{~mm})=5.0,20$ and 40 and $a$ high-speed video camera placed at $z D=60$. The two-phase flow images were taken at normal and micro gravity conditions for 10 seconds before and after the drop of the capsule started in the JAMIC drop tower.

\section{Apparatus}

The two-phase flow experiments were performed at normal and micro gravity conditions using an underground-drop shaft at the Japan Micro Gravity Center (JAMIC). The JAMIC drop shaft facility has high performances such as long free-fall depth (490 $\mathrm{m})$, long duration of microgravity (about $10 \mathrm{~s}$ ) and negligible residual gravity of $10^{-4} \sim 10^{-5}$ gN.

The flow loop was installed in a drop capsule of the JAMIC facility. The test section was a round pipe made of an acrylic resin. Its inner diameter, $D$, and length, $L$, were $9.0 \mathrm{~mm}$ and $630 \mathrm{~mm}$, respectively, and the $L D$ of the test section was 70 . Nitrogen gas was supplied by a gear pump and was introduced into a mixing chamber through a gas injector.

The flow measurements were performed by the image processing method with three digital video cameras placed at $z / D=5.0,20$ and 40 and a high-speed video camera placed at $z / D=60$. The two phase flow images were taken at normal and micro gravity conditions for 10 seconds before and after the drop of the capsule started. Due to the limited recording time of the high-speed video camera, the two-phase flow images at microgravity conditions were only taken at $z D=60$. The void fraction, interfacial area concentration, and bubble Sauter mean diameter were calculated from the obtained images with an assumption of a spheroidal bubble, and the bubble number density defined as the total number of bubbles per unit volume was simply determined by counting the number of bubbles.

\section{Result and discussion}

Figure 1 shows typical images of the axial flow developments at laminar and turbulent flow conditions. The bubble shape at microgravity conditions is almost spherical,

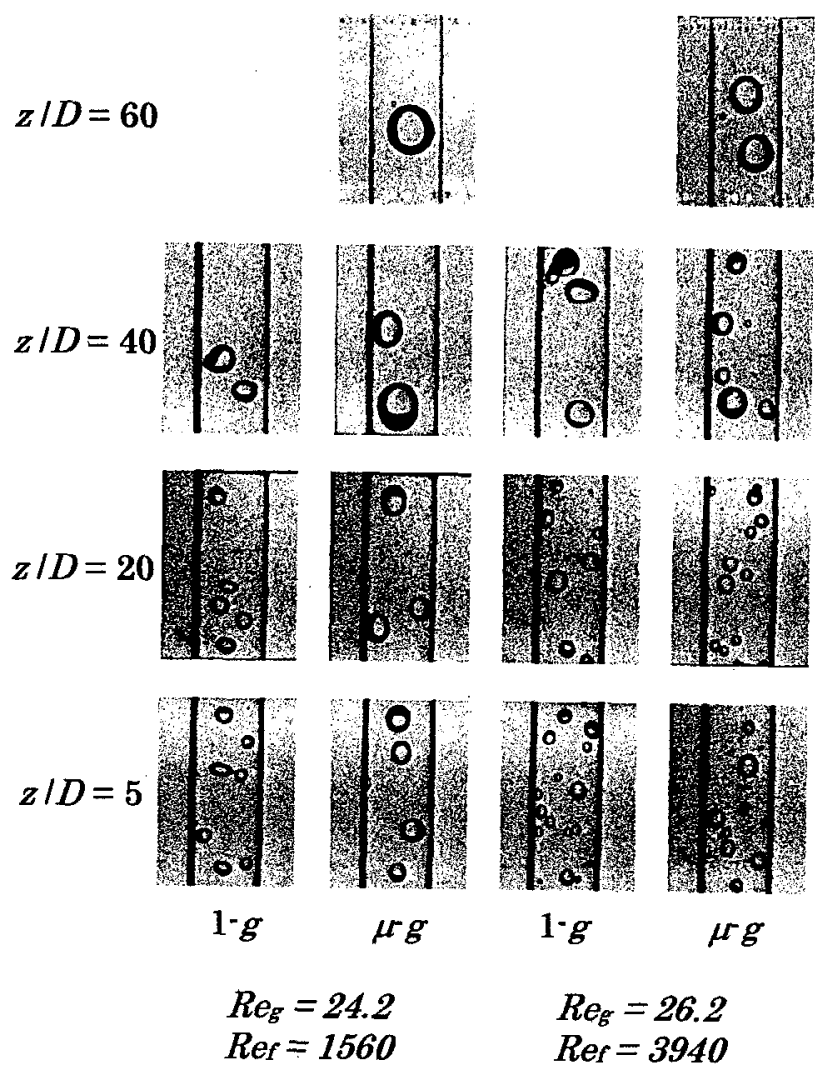

Fig.1 Images of axial developments of bubbly flow

whereas the bubble shape at normal gravity conditions is being deformed slightly with the increase in the liquid Reynolds number. Thus, the assumption of a spheroidal bubble may be a good approximation to measure the flow parameters. The significant bubble coalescence is observed at lower axial locations.

Figure 2 shows typical consecutive images of the bubble coalescence at microgravity conditions. The images clearly show that the following bubbles collide with the preceding ones resulting in the bubble coalescence. Thus, the bubble coalescence occurs even at low liquid Reynolds number and microgravity conditions where the coalescence due to the bubble random collision and the wake entrainment are generally considered to be negligible. The images also show that the moving velocities of some small bubbles are extremely low. These bubbles are considered to be sliding bubbles on the channel wall. It should be noted here that no bubble breakup 
$24 \mathrm{~ms}$
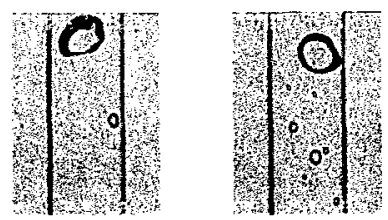

$16 \mathrm{~ms}$
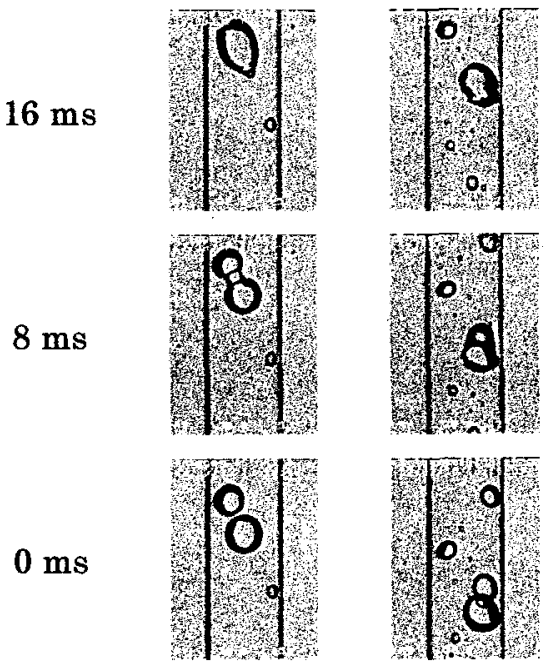

$$
R e_{\mathrm{g}}=24.1
$$

$R e_{\mathrm{g}}=26.2$

$R_{e \mathrm{r}}=1400$

$R e=3940$

Fig.2 Consecutive images of bubble coalescence process at $z D=60$

is visually observed in the tested experimental condition.

In order to understand the dominant mechanism of the bubble coalescence, a parameter named "dominant bubble interaction index" defined as Eq.(1) is introduced here.

$$
\xi \equiv \frac{n_{b, D}}{n_{b, U}}
$$

where $n_{\mathrm{b}, \mathrm{D}}$ and $\boldsymbol{a}_{\mathrm{b}, \mathrm{U}}$ are the bubble number density measured at downstream and upstream locations, respectively. Lower $\xi$ indicates more enhanced bubble coalescence, and $\xi<1$ or $\xi>1$ means that the dominant bubble interaction is bubble coalescence and breakup, respectively.

Figure 3 shows the effect of the liquid Reynolds number on the dominant bubble interaction parameter at the interval between $\not D=20$ and 40 . The figure clearly indicates that the dominant bubble interaction in the tested experimental conditions is bubble coalescence both at normal and micro gravity conditions, but the values of the index are almost constant with the liquid Reynolds number. The primary bubble coalescence mechanisms may be the bubble random collision, wake entrainment, and possibly velocity profile entrainment at laminar flow conditions. The contribution of these mechanisms to the bubble coalescence can be characterized as follows.

(1) The bubble random collision occurs due to the bubble random movement induced by the liquid turbulence. Thus, the increase in the liquid Reynolds number certainly enhances the bubble random collision. However, at very low liquid Reynolds number where the bubble random collision can be negligible, the increase in the liquid Reynolds number may not have significant impact on the bubble coalescence.

(2) The wake entrainment occurs due to the capture of the following bubble within a wake region of the preceding bubble. Since the wake entrainment is primarily

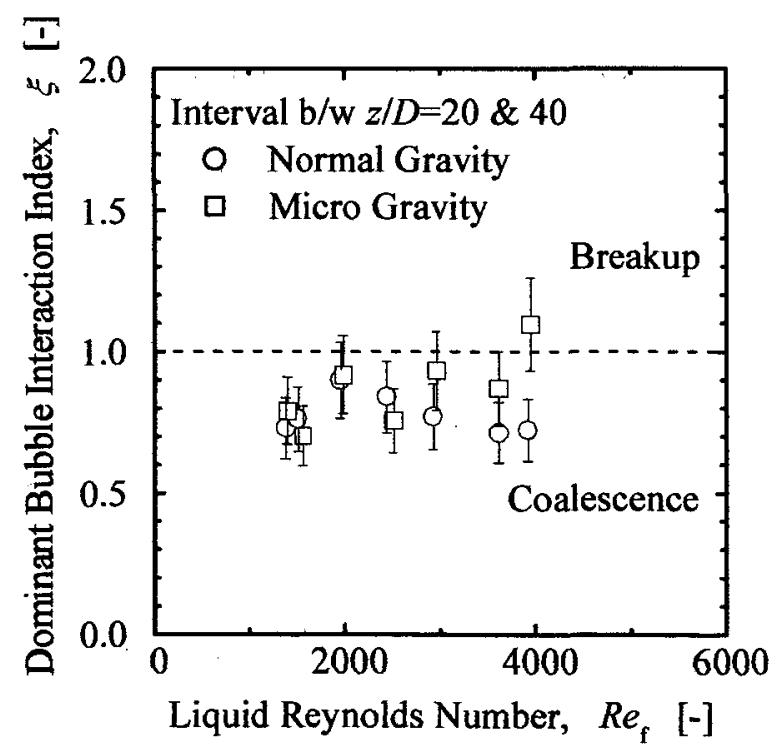

Fig.3 Dependence of dominant bubble interaction index on liquid Reynolds númber 
governed by the relative velocity between phases, the liquid Reynolds number may be insensitive to the wake entrainment. At microgravity conditions, the bubble coalescence due to the wake entrainment may be negligible. The wake entrainment at normal gravity conditions may be more pronounced at low liquid Reynolds number where the bubble random collision can be negligible.

(3) The velocity profile entrainment may occur at laminar flow conditions where the local liquid velocity at the channel center is much higher than that near the channel wall [4]. Although the introduction of bubbles into a liquid flow tends to flatter the liquid velocity profile, the velocity ratio between the center bubble and the sliding bubble is estimated to be $\mathbf{1 . 8 1}$ for the ideal parabolic liquid velocity profile at the conditions of $D=9 \mathrm{~mm}$ and $\angle D \mathrm{sm}_{\mathrm{m}}>=3$ $\mathrm{mm}$. The increase in the liquid Reynolds number reduces the velocity profile entrainment. The velocity profile entrainment at microgravity conditions may be more enhanced in comparison with that at normal gravity conditions, since negligible lift force acting on the bubbles tends to flatter the phase distribution resulting in high probability of the combinations of bubbles at the channel center and around the channel wall and bubble-induced turbulence tends to flatter the liquid velocity distribution.

Since the dominant mechanism indices at normal and micro gravity conditions are almost constant in the tested liquid Reynolds number conditions, the bubble random collision may be insignificant in the tested experimental conditions. The dominant mechanism index at microgravity conditions is similar to that at normal gravity conditions. This suggests the contribution of the velocity profile entrainment to the bubble coalescence at microgravity conditions is nearly equivalent to that of the wake entrainment to the bubble coalescence at normal gravity conditions.

\section{Conclusions}

In view of the great importance of two geometrical parameters such as void fraction and interfacial area concentration to the accurate two-phase flow analysis at microgravity conditions, axial developments of flow parameters such as void fraction, interfacial area concentration, bubble Sauter mean diameter, and bubble number density were measured in bubbly flow at microgravity and low liquid Reynolds number conditions where the gravity effect on the flow parameters were pronounced. The measurements were also performed in the similar flow range at normal gravity conditions. The transport mechanisms of the flow parameters were discussed based on the data measured at normal and microgravity conditions in detail and the drift-flux model developed at microgravity conditions were compared with the measured data.

\section{References}

[1] Tomiyama, A., Kataoka, I., Zun, I., Sakaguchi, T.: Drag coefficients of single bubbles under normal and micro gravity conditions, JSME Int. J. B-41 (1998) 472-479.

[2] Hibiki, T., Ishii, M.: One-dimensional drift-flux model and constitutive equations for relative motion between phases in various two-phase flow regimes, Int. J. Heat Mass Transfer, 46 (2003) 4935-4948.

[3] Hibiki, T., Takamasa, T., Ishii, M.: One-dimensional drift-flux model and constitutive equations for relative motion between phases in various two-phase flow regimes at microgravity conditions, Proc. 12th Int. Conf. Nucl. Eng., Arlington, USA, Paper Num.: ICONE-12-49037 (2004).

[4] Takamasa, T., Iguchi, T., Hazuku, T., Hibiki, T., Ishii, M.: Interfacial area transport of bubbly flow under microgravity environment, Int. J. Multiphase Flow 29 (2003) 291-304. 